\title{
Carbon-rich monolayers on ITO as highly sensitive platforms for detecting polycyclic aromatic hydrocarbons in water: The case of
} pyrene

\author{
Jose Muñoz, Núria Crivillers and Marta Mas-Torrent ${ }^{\star}$ \\ Institut de Ciencia de Materials de Barcelona (ICMAB-CSIC) and CIBER-BBN \\ Campus de la UAB, 08193 Bellaterra (Cerdanyola del Vallès), Spain \\ E-mail:mmas@icmab.es
}

\begin{abstract}
The determination of polycyclic aromatic hydrocarbons (PAHs) in water at low levels is a current challenge given their great impact on the health and safety of the public. Here, a novel pyrene-based self-assembled monolayer (SAM) platform is exploited as PAH sensing recognition device. Interestingly, the formation of $\pi-\pi$ sandwich complexes between PAHs and the recognition element switches the surface electron transfer capability. The unique supramolecular interaction between identical aromatic molecules provides a highly sensitive and selective sensor for pyrene in the order of part per trillion. Accordingly, and using pyrene as a proof-of-concept, this work presents the basis for an 'at-point-of-use' impedimetric sensor focused on a highly sensitive carbon-rich SAM for PAHs determination in water at ultra-trace levels.
\end{abstract}

Polycyclic aromatic hydrocarbons (PAHs), such as pyrene, naphthalene, anthracene, fluoranthene, benzo[a]pyrene and benzo[ghi]perylene, are a class of organic environmental pollutants mainly formed in natural or manmade incomplete combustion processes of fossil fuels, tobacco, solid waste and cooking foods. ${ }^{[1],[2],[3]}$ PAHs possess a potential health hazard because of their carcinogenic, mutagenic as well as teratogenic properties. ${ }^{[4],[5]}$ Owing to their alarming abundance in the environment and adverse health effects, the US Environmental Protection Agency (EPA), the European Union (EU) and the International Agency for Research on Cancer (IARC) have promulgated several PAH compounds in their lists of priority pollutants in surface water for drinking purposes. ${ }^{[6],[7],[8]}$ For example, EPA's maximum contaminant level $(\mathrm{MCL})$ for PAHs in drinking water is 0.2 ppb. Further, due to the high hydrophobicity of PAHs, their solubility in water is very low, and thus their determination in water samples becomes extremely challenging. Under this context, rapid and sensitive analytical methodologies for PAHs sensing at low concentrations are mandatory.

To date, the standard methods for PAH determination are mainly based on bench-top laboratory instruments, such as high performance liquid chromatography coupled to fluorescence detectors, ${ }^{\left[{ }^{[0]}\right.}$ gas chromatography with mass spectrometry, ${ }^{[0]}$ and capillary electrophoresis. ${ }^{[1]}$ Usually, in order to increase the analyses sensitivity these methods require a lengthy and laborious preconcentration and extraction steps, which hinders their use for in-field analysis. Alternative spectroscopic techniques, as the surfaceenhanced Raman spectroscopy (SERS) ${ }^{[12]}$ and the fluorescence spectroscopy ${ }^{[13]}$ have emerged for the in situ PAH identification. Very few electrochemical platforms, mainly based on voltammetric bio-sensors, have been developed to detect different PAHs due to the trace concentration of these pollutants in water. ${ }^{[14]}$ These devices are basically focused on using electroactive bio-receptors for immunosensing, for example, benzo[a]pyrene. ${ }^{[15],[16],[17]}$ However, the impedimetric sensing of PAHs still remains an unexplored field.

The covalent grafting of functional molecules on solid supports has been shown to be a promising route to fabricate smart switchable surfaces that respond to external stimuli (i.e., light, electrical, chemical, etc). ${ }^{[18],[19],[20],[21],[22]}$ Accordingly, and motivated by the possibility to develop a novel highly sensitive electrochemical platform for PAHs determination, a hybrid-material based on a selfassembled monolayer (SAM) of an aromatic carbon molecule on Indium Tin Oxide (ITO) able to interact with PAHs has been fabricated. Pyrene was used as a probe recognition agent owing to its planar aromatic structure, which allows synergistic $\Pi-\Pi$ interactions with PAHs. ${ }^{[23]}$ Importantly, owing the unique supramolecular interactions between identical aromatic species, a highly selective and sensitive pyrene sensor was realized. This work advocates that the combination of using a single active molecular monolayer with electroanalysis, and concretely impedimetric analysis, can be a viable, fast, integrable alternative to determine PAHs in water. Further, this methodology could be easily extended to different types of carbon-based pollutants through the strategically inclusion of specific carbon-based recognition groups by surface engineering.

Following Scheme 1, the recognition agent was attached on ITO film coated glass (S1) via a three-step process (see experimental part from SI). Briefly, $\mathbf{S} 1$ surfaces $\left(6 \times 25 \mathrm{~cm}^{2}\right)$ were cleaned with different organic solvents of increasing polarity and then activated to induce the formation of hydroxyl groups in an oxidizing bath of $\mathrm{NH}_{4} \mathrm{OH}: \mathrm{H}_{2} \mathrm{O}_{2}: \mathrm{H}_{2} \mathrm{O}(1: 1: 5)$ at $80{ }^{\circ} \mathrm{C}$ for 30 min and rinsed thoroughly with plenty of water (step i)). The resulting activated-S1 substrate was immersed in a toluene solution of 3glycidoxypropyltrimethoxy-silane $(1 \% \mathrm{v} / \mathrm{v})$ at $60^{\circ} \mathrm{C}$ for $12 \mathrm{~h}$, resulting in the SAM-based ITO-silane terminated with an epoxide group (S2, step ii)). Finally in step iii) $\mathbf{S} 2$ was immersed in a $1 \mathrm{mM}$ solution of 1-aminopyrene in toluene at $80^{\circ} \mathrm{C}$ for $36 \mathrm{~h}$ forming a hydroxyl and a secondary amine (ITO-silane-pyrene, S3). Such hybrid-material was then exploited as the SAM-based recognition platform for PAH electroanalysis.

The successful fabrication of the novel recognition platform was confirmed by static water contact angle, cyclic voltammetry (CV) and electrochemical impedance spectroscopy (EIS), as it is shown in Figure 1. Further surface characterization data by means of Xray photoelectron spectroscopy (XPS) measurements is available in Figure S1. 


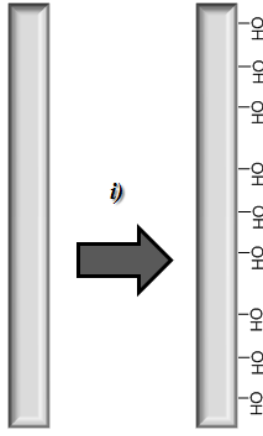

S1

Activated-S1

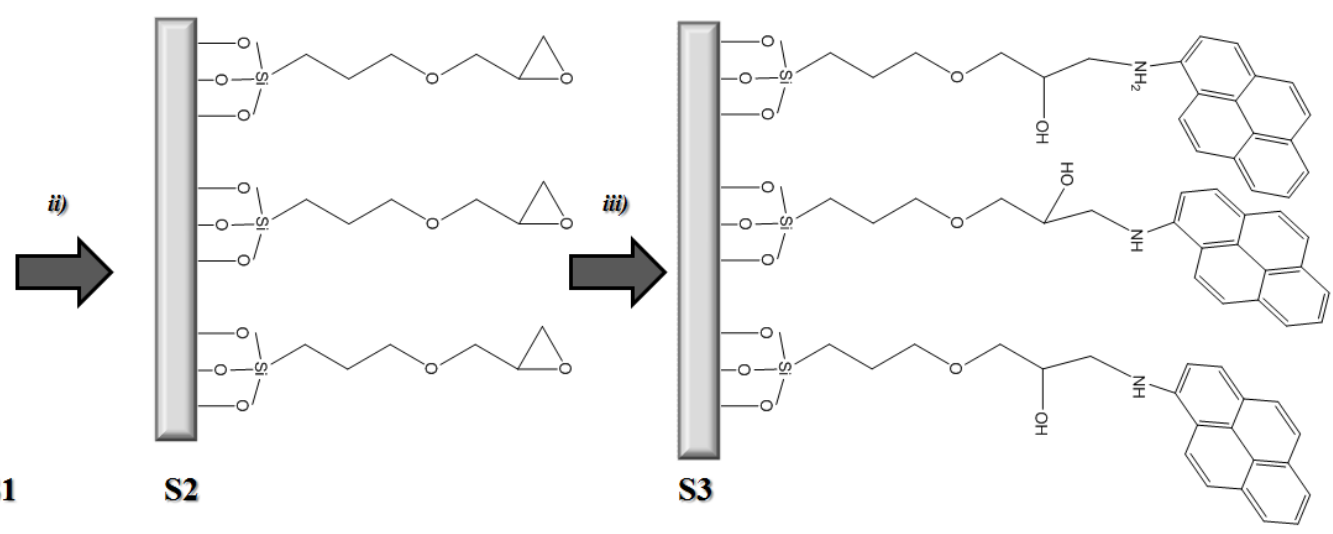

Scheme 1. Schematic representation of the three SAM immobilization steps on the ITO electrode for sensor fabrication. i) Oxidizing bath $\mathrm{NH}_{4} \mathrm{OH}: \mathrm{H}_{2} \mathrm{O}_{2}: \mathrm{H}_{2} \mathrm{O}$ (1:1:5), ii) Immersion the activated-S1 substrate in a toluene solution containing $1 \%$ (v/v) of 3-glycidoxypropyltrimethoxysilane. iii) Immersion of S2 in a toluene solution containing $1 \mathrm{mM}$ of 1 -aminopyrene at $80^{\circ} \mathrm{C}$ for $36 \mathrm{~h}$.

The wettability changes of the ITO surfaces at different functionalization stages were measured by contact angle at room temperature, using water as solvent. Figure 1a reveals that the static contact angle decreases significantly from $82.9 \pm 0.1^{\circ}$ (S1) to $62 \pm 4^{\circ}$ (S2) after the epoxy SAM incorporation. When the pyrene-based recognition agent was incorporated, the wettability of the surfaces was reversed, increasing to $74 \pm 4^{\circ}$ (S3) due to the more hydrophobic character of the SAM. Electrochemical experiments were carried out in a three-electrode configuration cell, using a Pt wire and an $\mathrm{Ag} / \mathrm{AgCl}$ (saturated $\mathrm{KCl}$ ) electrode as the auxiliary and the reference electrodes, respectively, and the ITO-based substrates as the working electrodes. The electrolyte consisted of a $0.1 \mathrm{M}$ $\mathrm{KCl}$ aqueous solution containing $10 \mathrm{mM}\left[\mathrm{Fe}(\mathrm{CN})_{6}\right]^{3-14-}$ as a benchmark redox marker since it is very sensitive to the electrode surface characteristics. ${ }^{[24]}$

As it is depicted in Figure $\mathbf{1}(\mathbf{b}, \mathbf{c}), \mathrm{CV}$ and EIS were used to monitor all the functionalization steps of the ITO-based surfaces. Cyclic voltammograms from Figure 1b shows a progressive peak-to-peak separation $(\Delta E)$ enhancement from S1 to S2 and S3, indicating lower system reversibility. Additionally, this effect is accompanied with a decrease in the redox peak intensity $\left(I_{p}\right)$ because of the insulating properties of the attached molecules on the $\mathbf{S 1}$ surface, fact that inhibits the electronic transmission of the electrode. The electronic transfer properties were also evaluated by EIS. As it is depicted in Figure 1c, EIS analysis were represented by Nyquist plots (real impedance, $Z_{r}$ vs. imaginary impedance, $Z_{i}$ ) and modeled by fitting the data with a typical Randles equivalent circuit: $R_{s} \cdot\left(C_{d l} \cdot\left[R_{c t} \cdot Z_{w l}\right]\right)$; where $R_{s}$ represents the solution resistance and depends on the electrolyte nature, $C_{d l}$ is the double-layer capacitance and it is directly related to charging and background current, $Z_{w}$ is the Warburg impedance, which arises from masstransfer limitations, and $R_{\mathrm{ct}}$ is the charge transfer resistance, which is inversely related to the electron transfer rate. ${ }^{[25]}$

$\mathrm{R}_{\mathrm{ct}}$ was determined by the semicircle diameter of $Z_{r}$. Such resistance was much larger in $\mathbf{S 3}(170.2 \Omega)$ than in S2 (47.9 $\Omega$ ), which in turn was larger than the one found for $\mathbf{S 1}(22.8 \Omega)$. These results are in agreement with the fact that the SAM layers hinder the electron transfer from the redox probe to the electrode surface. These changes also indicate that both SAM surface modification steps were successfully achieved.

The electrochemical properties and stability of the SAM-based recognition agent (S3) were also investigated using a $0.5 \mathrm{M} \mathrm{H}_{2} \mathrm{SO}_{4}$ solution containing $0.1 \mathrm{M} \mathrm{KCl}$ as the electrolyte. As it is depicted in Figure $\mathbf{S} 2 \mathbf{A}$, a pair of redox peaks was observed at $E^{1 / 2}=0.35 \mathrm{~V}$ (vs. $\mathrm{Ag} / \mathrm{AgCl}$ at scan rate of $50 \mathrm{mV} \cdot \mathrm{s}^{-1}$ ), which are associated with the redox process of the derived dione species, which is the most stable oxidation product of the pyrene ring system that is formed under these conditions. ${ }^{[26]}$ The almost insignificant voltage difference between the anodic and catodic peak of $36 \mathrm{mV}$ is characteristic of surface-confined molecules, which was further confirmed by the linear dependence of the $I_{p}$ on the scan rate $(\mathrm{u})$ in the range of $50-1000 \mathrm{mV} \cdot \mathrm{s}^{-1}$. A surface coverage of $6.3 \times 10^{-12} \mathrm{~mol} \cdot \mathrm{cm}{ }^{-2}$ was estimated by integration of the anodic peak. To explore the stability of the SAM, the S3 platform was subjected to 100 voltammetric cycles at $\mathrm{u}=1 \mathrm{~V} \cdot \mathrm{s}^{-1}$ (see Figure S2 B) observing practically no decrease in the measured current and, hence, pointing out the high stability of the developed system.

Having verified the successful preparation of the novel S3 recognition platform, this substrate was used as working electrode for sensing purposes, using pyrene as a model PAH analyte. For this aim, the working electrodes were dipped in an aqueous solution with the desired concentration of PAHs. The incubation took place for $15 \mathrm{~min}$. Then, the sensor was washed with water and incorporated in the electrochemical cell. Pyrene was monitored in terms of impedimetric measurements, following the electrostatic and/or steric barrier presented to the $\left[\mathrm{Fe}(\mathrm{CN})_{6}\right]^{3 / 4-}$ redox probe at the recognition system surface (see Nyquist plots of Figure 2a). It should be noticed that in the working conditions (i.e., neutral media and bias voltage $+150 \mathrm{mV}$, see SI for experimental details) no prussian blue is generated when using this redox probe that could affect the sensing response and that the silane-ITO monolayers are stable. ${ }^{[27]}$ 
(a)
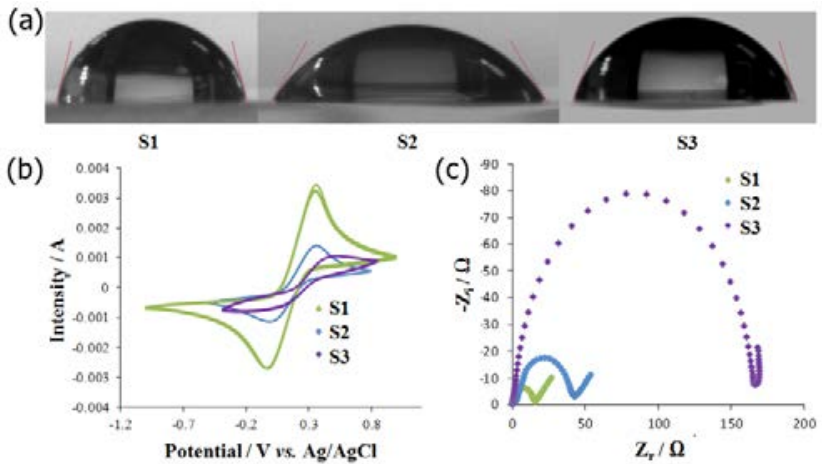

Figure 1. (a) Static water contact angles of $\mathbf{S} 1, \mathbf{S} 2$ and $\mathbf{S} 3$ and their electrochemical characterization by (b) $\mathrm{CV}$ (scan rate: $50 \mathrm{mV} \cdot \mathrm{s}^{-1}$ ) and (c) EIS (freq. range: $100 \mathrm{kHz}-100 \mathrm{mHz}$; bias potential: $+150 \mathrm{mV}$ and $\mathrm{AC}$ amplitude: $5 \mathrm{mV}$ ). Experiments in $\mathrm{b}$ and c were carried out using a $0.1 \mathrm{M} \mathrm{KCl}$ aqueous solution containing 10 $\mathrm{mM}\left[\mathrm{Fe}(\mathrm{CN})_{6}\right]^{3-14-}$

The sensing response is determined by the $\Pi-\Pi$ supramolecular interactions between the recognition agent attached on the electrode surface and the PAH analyte forming a sandwich complex. ${ }^{[28]}$ Further, it should be highlighted that a limit of detection as low as $1.75 \pm 0.06 \mathrm{ppt}$ for pyrene was realized (see Figure $\mathbf{2 b}$ ). Compared with a recent pyrene-based recognition material based on the fluorescence response, the limit of detection obtained for pyrene by the presented S3 impedimetric platform is > 1000 times lower (from $2 \mathrm{ppb}$ to $1.75 \mathrm{ppt}$ ). ${ }^{[29]}$ This can be ascribed to the fact that the active sensing layer here is only formed by one single molecular monolayer and hence, any small surface modification is greatly translated into an detectable output signal. This fact in synergy with EIS technique offers several advantages to this device towards PAHs determination, due to its much higher sensitivity and faster response combined with a simple detection means.

The adsorption of molecules on the electrode surface commonly leads to an insulating layer that retards the interfacial electron transfer kinetics between the redox probe and the electrode. ${ }^{[30]}$ From an electrochemical point of view, this fact generates an increase on the $\mathrm{R}_{\mathrm{ct}}$ value. Blank reference tests with the bare $\mathbf{S 1}$ surface and using pyrene concentrations 1000 times larger than the ones employed for the sensing, experiments, revealed this tendency (see Figure S3). In contrast when S3 was used, it was observed that the $\pi-\pi$ interactions lead to a linear decrease (rather than the common increase) in the $R_{c t}$ value (Figure $\mathbf{2 a}$ ).
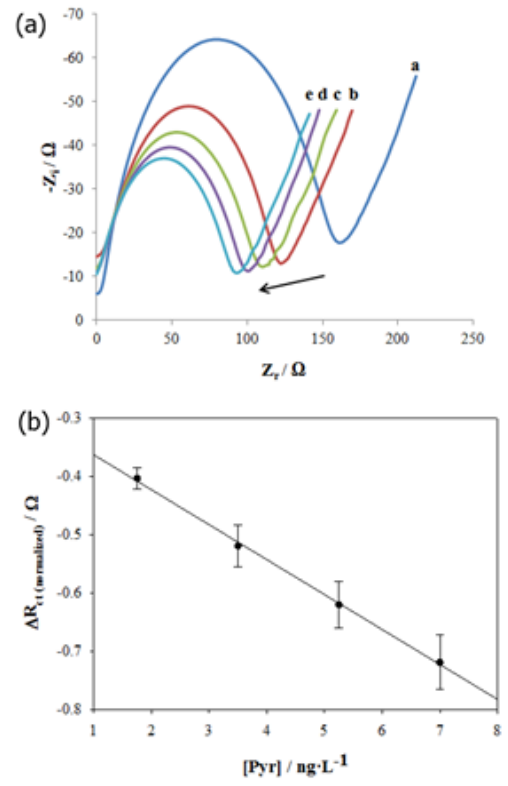

Figure 2. (a) Impedance sensing of pyrene recorded at the $\mathbf{S} 3$ electrode in the presence of increasing concentrations of pyrene: a) 0 , b) 1.75 , c) 3.50 , d) 5.25 and e) 7.00 ppt. (b) Calibration plot representing $\Delta R_{c t(n o r m a l i z e d) ~}=\Delta R_{c t} / R_{0}$ vs. pyrene concentration ([Pyr]), with their corresponding error bars $(n=9)$. Measurements obtained after 15 min incubation with the pyrene solution.

As it is illustrated in Figure 3a, such behavior can be accounted by the superb supramolecular interactions between recognition agent and planar PAHs carbon molecules (e.g. pyrene) that provide a molecular reorganization within the SAM. This fact induces the formation of empty channels leaving more exposed the electrode surface, where the $\left[\mathrm{Fe}(\mathrm{CN})_{6}\right]^{3-14-}$ redox marker can penetrate facilitating its redox reaction (Figure 3c). In this way, the resistance to electron transfer is decreased, switching the initial impedance value (Figure 3d). Accordingly, the same trend in impedimetric switching was observed when using anthracene and benzo[ghi]perylene as alternative PAH analytes (see Figure S4). To further verify this, a model non-planar aromatic carbon-based molecule as fullerene was also tested as analyte in a concentration of 0.035 ppt (Figure S5). As shown in Figure 3b, fullerenes block the electrode surface and the interfacial electron transfer reaction is hindered and therefore, an $R_{c t}$ increase is observed (Figure $3 c$ - 
d). Interestingly, these electrochemical results reveal that the developed recognition system is able to differentiate between planar and non planar carbon-based aromatic molecules.

The specificity of the method for pyrene determination compared to other PAHs was investigated using this methodology and a $[\mathrm{PAH}]=7.00 \mathrm{ppt}$ for each analyte (Figure S6). As it is depicted in Figure 4, a much higher response to pyrene, which contains 4 aromatic rings, regarding to other PAHs, such as napththalene, anthracene, benzo[a]pyrene and benzo[ghi]perylene with 2, 3, 5 and 6 aromatic rings, respectively, was achieved. Likewise, when fluoranthene was used as an alternative PAH analyte containing 4 aromatic rings a lower impedimetric response was obtained. This is attributed to the most favorably supramolecular interactions that take place between identical aromatic molecules. ${ }^{[23]}$ In this sense, since the SAM-based recognition element employed for PAH analysis is the pyrene, the developed $\mathbf{S} 3$ platform is the most favorable system for sensing selectively pyrene.

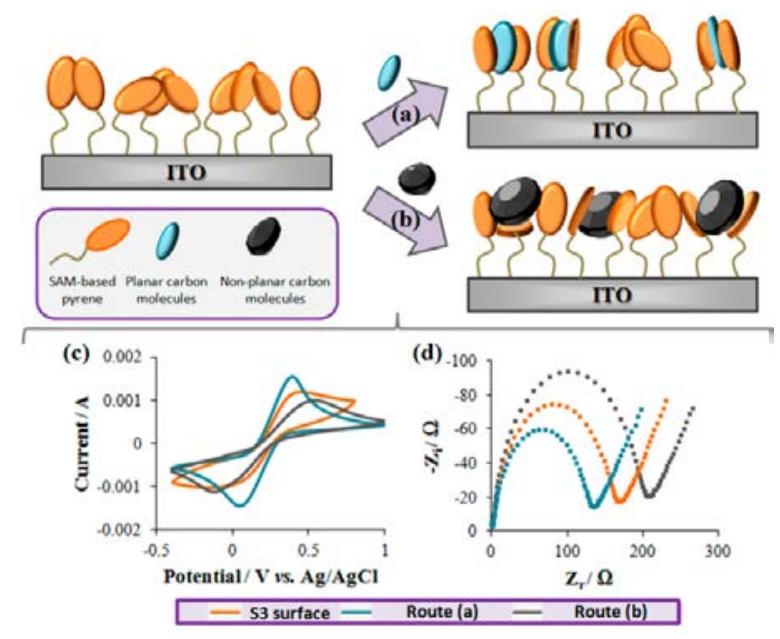

Figure 3. Schematic illustration of the supramolecular recognition of $\mathbf{S} 3$ towards (a) planar and (b) non-planar carbon molecules. Representative electrochemical performance by means of CV (c) and EIS (d) of the pyrene SAM S3 initially and after interaction with pyrene (Route a) and fullerene (Route b).

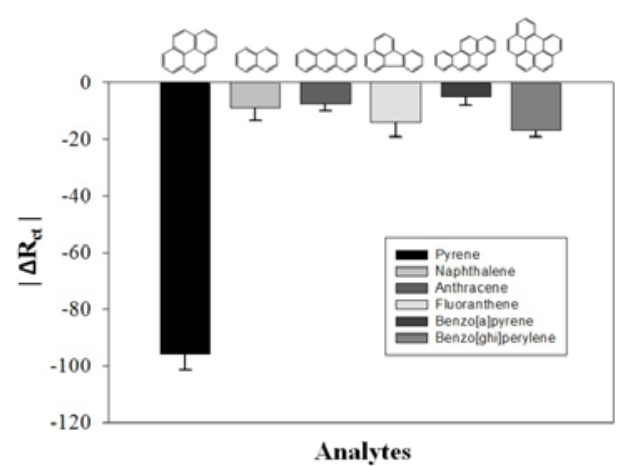

Figure 4. Bar-plot with their corresponding error bars $(n=3)$ representing the specificity of the developed SAM-based impedimetric recognition platform towards different PAHs in water at a concentration of 7.00 ppt.

Additionally, a complex mixture solution containing $7 \mathrm{ppt}$ of the aforementioned PAH interferences together with the same concentration of the pyrene analyte was also investigated, recovering the $90.4 \%$ of the pyrene signal (see Figure S6). These results clearly demonstrate the excellent performance and selectivity of the developed pyrene-based SAM platform towards the determination of pollutant pyrene in water, even in complex matrices. It can also be envisaged that tailored sensing platforms with different aromatic hydrocarbon-based recognition molecules could be potentially designed for recognizing different target PAHs, making it possible to develop selective platforms towards the specific PAH target.

In conclusion, an ultrasensitive carbon-rich SAM platform has been developed for the simple, rapid and sensitive electrochemical determination of PAHs in water. This device could be easily miniaturized since the transduction method is electronic. In particular, electrochemical impedance spectroscopy has been employed to analyse such pollutant compounds. As a proof-of-concept, pyrene was used as recognition agent. The electrochemical method of the pyrene-based sensing platform is based on the excellent $\pi-\pi$ supramolecular interactions that are formed between PAH and the SAM. This fact yields to sense PAHs at part per trillion levels (in the order of $\mathrm{pM}$ concentrations). An excellent selectivity for pyrene with a detection limit as low as $1.75 \pm 0.06$ ppt was achieved thanks to its identical molecular structure with the recognition element, which enhances the formation of $\pi-\pi$ sandwich assemblies. Interestingly, it has been demonstrated that the developed sensor is able to impedimetrically distinguish planar aromatic molecules from the non-planar ones depending on the type of recognition-induced switching of electron charge transfer. Finally, this work demonstrates that the synergetic combination of surface engineering employing nanostructured carbon materials with impedimetric spectroscopy is a promising strategy for the development of a novel generation of environmental sensors for monitoring several pollutant carbon-based nanomaterials in water at ultra-trace levels. 


\section{Acknowledgements}

This work was funded by the ERC StG 2012-306826 e-GAMES. The authors also thank the ITN iSwitch 642196 project, Networking Research Center on Bioengineering, Biomaterials, and Nanomedicine (CIBER-BBN), the DGI (Spain) project FANCY CTQ201680030-R, the Generalitat de Catalunya (2014-SGR-17) and the Spanish Ministry of Economy and Competitiveness, through the "Severo Ochoa" Programme for Centers of Excellence in R\&D (SEV-2015-0496).

\section{References}

[1] M. T. Alcántara, J. Gómez, M. Pazos, M. A. Sanromán, J. Hazard. Mater. 2009, 166, 462-468.

[2] K.-H. Kim, S. A. Jahan, E. Kabir, R. J. Brown, Environ. Int. 2013, 60, 71-80.

[3] H. I. Abdel-Shafy, M. S. Mansour, Egypt. J. Pet. 2016, 25, 107-123.

[4] R. Wang, G. Liu, J. Zhang, SSci. Total Environ. 2015, 538, 180-190.

[5] T. Rengarajan, P. Rajendran, N. Nandakumar, B. Lokeshkumar, P. Rajendran, I. Nishigaki, Asian. Pac. J. Trop. Biomed. 2015, 5, 182-189.

[6] S. A. Bortolato, J. A. Arancibia, G. M. Escandar, Anal. Chem. 2008, 80, 8276-8286.

[7] E. Union: Directive 2013/39/EU of the European parliament and of the council of 12 August 2013 amending Directives 2000/60/EC and 2008/105/EC as regards priority substances in the field of water policy, Official Journal of the European Union 2013.

[8] E. Manoli, A. Kouras, O. Karagkiozidou, G. Argyropoulos, D. Voutsa, C. Samara, Environ. Sci. Pollut. Res. Int. 2016, 23, $3556-3568$.

[9] M. Fernández-Amado, M. C. Prieto-Blanco, P. López-Mahía, S. Muniategui-Lorenzo, D. Prada-Rodríguez, Anal. Chim. Acta 2016, $906,41-57$.

[10] J. I. Cacho, N. Campillo, P. Viñas, M. Hernández-Córdoba, Food Chem. 2016, 190, 324-330

[11] L. Ferey, N. Delaunay, D. N. Rutledge, C. B. Cordella, H. This, A. Huertas, Y. Raoul, P. Gareil, Talanta 2014, 119, 572-581.

[12] J. Du, J. Xu, Z. Sun, C. Jing, Anal. Chimi. Acta 2016, 915, 81-89.

[13] L. Greene, B. Elzey, M. Franklin, S. O. Fakayode, Spectrochim. Acta, Part A 2017, 174, 316-325.

[14] M. Díaz-González, M. Gutiérrez-Capitán, P. Niu, A. Baldi, C. Jiménez-Jorquera, C. Fernández-Sánchez, TrAC, Trends Anal. Chem. 2016, 77, 186-202.

[15] G. Lux, A. Langer, M. Pschenitza, X. Karsunke, R. Strasser, R. Niessner, D. Knopp, U. Rant, Analy. Chem. 2015, 87, 4538-4545.

[16] Y. Ni, P. Wang, H. Song, X. Lin, S. Kokot, Anal. Chim. Acta 2014, 821, 34-40.

[17] A. Ahmad, E. Moore, Analyst 2012, 137, 5839-5844.

[18] E. Marchante, N. Crivillers, M. Buhl, J. Veciana, M. Mas-Torrent, Angew. Chem. Int. Ed. 2016, 55, 368-372.

[19] I. Alcón, M. Gonidec, M. Ajayakumar, M. Mas-Torrent, J. Veciana, Chem. Sci. 2016, 7, 4940-4944.

[20] T. Gupta, M. E. Van Der Boom, Angew. Chem. Int. Ed. 2008, 47, 5322-5326.

[21] M. Mas-Torrent, C. Rovira, J. Veciana, Adv. Mater. 2013, 25, 462-468.

[22] Z. Liu, A. A. Yasseri, J. S. Lindsey, D. F. Bocian, Science 2003, 302, 1543-1545.

[23] S. Liu, M. Wei, X. Zheng, S. Xu, C. Zhou, Anal. Chim. Acta 2014, 826, 21-27.

[24] J. Muñoz, F. Céspedes, M. Baeza, J. Electrochem. Soc. 2015, 162, B217-B224.

[25] J. Muñoz, L. J. Brennan, F. Céspedes, Y. K. Gun'ko, M. Baeza, Compos. Sci. Technol. 2016, 125, 71-79.

[26] M. Mazur, G. Blanchard, J. Phys. Chem. B 2004, 108, 1038-1045.

[27] S. Vogt, Q. Su, C. Gutiérrez-Sánchez, G- Nöll, Anal. Chem. 2016, 88, 4383-4390.

[28] K. Yang, L. Zhu, B. Xing, Environ. Sci. Technol. 2006, 40, 1855-1861.

[29 B. D. B. Tiu, R. J. Krupadam, R. C. Advincula, Sens. Actuators, B 2016, 228, 693-701.

[30] M. C. Rodriguez, A.-N. Kawde, J. Wang, Chem. Comm. 2005, 4267-4269. 